\title{
COPPER NITRATE TRIHYDRATE CATALYZED EFFICIENT ONE POT SYNTHESIS OF AMIDOALKYLNAPHTHOL DERIVATIVES.
}

\section{AAYESHA NASREEN,* RITA M. BORIK}

Department of Chemistry, College of Sciences (Girls), Jazan University, 6811-Arroudah

P.O Box No. 2097, Jazan 82724-3750, Saudi Arabia, Fax: +966 073227066

\begin{abstract}
:
In the present study we extend the scope of the $\mathrm{Cu}\left(\mathrm{NO}_{3}\right)_{2} \cdot 3 \mathrm{H}_{2} \mathrm{O}$ catalyzed synthesis of amido alkyl naphthols and the results are presented here. In order to optimize the reaction conditions initially we studied the efficacy of $\left.\mathrm{Cu}_{(\mathrm{NO}}\right)_{2}$. $3 \mathrm{H}_{2} \mathrm{O}$ by taking catalytic amount of $10 \mathrm{~mol} \%$ and benzaldehyde (1 mmol) $\beta$-naphthol (1 mmol) and acetamide $(1.2 \mathrm{mmol})$ in acetonitrile $(10 \mathrm{ml})$ as model reaction. We have developed a practical and new, general efficient procedure for the one pot synthesis of amidoalkyl naphthols by coupling of various aromatic aldehydes with amides urea and $\beta$-naphthol using $\mathrm{Cu}\left(\mathrm{NO}_{3}\right)_{2} 3 \mathrm{H}_{2} \mathrm{O}$ as catalyst. The present protocol has several advantages of readily available, inexpensive catalyst, mild reaction conditions, easy handling, excellent yields, greater selectivity, operational and experimental simplicity. We believe that $\mathrm{Cu}\left(\mathrm{NO}_{3}\right)_{2} \cdot 3 \mathrm{H}_{2} \mathrm{O}$ catalyzed methodology will definitely be a valuable addition to the existing process in the field of amidoalkyl naphthols.
\end{abstract}

Keywords: Amidoalkyl naphthols, $\beta$-naphthol, Multi component reaction, $\mathrm{Cu}\left(\mathrm{NO}_{3}\right)_{2} \cdot 3 \mathrm{H}_{2} \mathrm{O}$.

\section{INTRODUCTION}

Multicomponent reactions (MCRs) have been proved to be a very elegant and rapid way to access complex structures in a single synthetic operation from simple building blocks, and show high atom-economy, high selectivity and procedural simplicity due to the formation of carboncarbon and carbon-heteroatom bonds in one-pot and generally afford good yields. ${ }^{[1]}$ Multi-component reactions have attracted considerable attention in organic synthesis as they can produce target products in a single operation without isolating the intermediates and thus reducing the reaction times and energy input. ${ }^{[2,3]}$

Compounds bearing 1,3-amino-oxygenated functional motifs are common in a variety of biologically important natural products and potent drugs, including a number of nucleoside antibiotics and HIV protease inhibitors, such as ritonavir and lipinavir. ${ }^{[4]}$ Moreover, 1 -amidoalkyl naphthol can be easily hydrolyzed to 1-aminoalkylnaphthol, which shows biological activities like hypotensive, bradycardiac effect and catalyst in asymmetric synthesis. ${ }^{[5,6]}$

The importance of amidoalkyl naphthols for their synthesis has attracted renewed attention and various improved procedures have been reported. Several alternative and efficient methods have been developed for the synthesis of amidoalkylnaphthol derivatives by multi component reaction of $\beta$-naphthol, aldehyde and amide in the presence of different catalysts such as Montmorillonite K10clay, ${ }^{[7]}$ $\mathrm{Ce}\left(\mathrm{SO}_{4}\right)_{2},{ }^{[8]} \quad \mathrm{K}_{5} \mathrm{CoW}_{12} \mathrm{O}_{40} \cdot 3 \mathrm{H}_{2} \mathrm{O},{ }^{[9]} \quad \mathrm{p}-\mathrm{TSA},{ }^{[10]}$ Sulfamic acid/ultrasound, ${ }^{[11]}$ Ionic liquids, ${ }^{[12]}$ Indion-130, $\left.{ }^{[13]} \quad \mathrm{Al}\left(\mathrm{H}_{2} \mathrm{PO}_{4}\right)_{3},{ }^{[14]} \quad \mathrm{Fe}\left(\mathrm{HSO}_{4}\right)_{3}\right)^{[15]}$ $\mathrm{Yb}(\mathrm{OTf})_{3},{ }^{[16]}$ Wetcyanuric chloride, ${ }^{[17]} \quad \mathrm{Al}_{2} \mathrm{O}_{3}$ $\mathrm{HClO}_{4}{ }^{[18]}$ Silica chloride $\left(\mathrm{SiO}_{2}-\mathrm{Cl}\right) /$ ultrasound, ${ }^{[19]}$ indium(III)chloride, ${ }^{[20]} \quad \mathrm{Sr}(\mathrm{OTf})_{2},{ }^{[21]} \quad \mathrm{P}_{2} \mathrm{O}_{5},{ }^{[22]}$ $\mathrm{NaHSO}_{4} \cdot \mathrm{SiO}_{2}{ }^{[23]} \quad \mathrm{H}_{4} \mathrm{SiW}_{12} \mathrm{O}_{40}{ }^{[24]}$ Zeolite $\mathrm{H}$ $\mathrm{BEA},{ }^{[25]} \quad \mathrm{N}, \mathrm{N}, \mathrm{N}^{\prime}, \mathrm{N}^{\prime}$-Tetrabromobenzene1,3-disulfonamide(TBBD), ${ }^{[26]} \quad \mathrm{KHSO}_{4}{ }^{[27]}$ tritylchloride ${ }^{[28]} \quad$ Bismuth(III) nitrate pentahydrate, ${ }^{[29]} \quad \mathrm{H}_{3} \mathrm{BO}_{3}{ }^{[30]} \quad \mathrm{Mg}\left(\mathrm{ClO}_{4}\right)_{2}{ }^{[31]}$ MNPs- $\mathrm{SO}_{3} \mathrm{H},{ }^{[32]}$ and Succinic acid. ${ }^{[33]}$ In which some of often involves the use of expensive reagents, hazard solvents and tedious workup.

$\mathrm{Cu}\left(\mathrm{NO}_{3}\right)_{2} \cdot 3 \mathrm{H}_{2} \mathrm{O}$ has been extensively used as a mild catalyst for a variety of organic trans formations. ${ }^{[34,35,37-40]}$ In continuation of our work to develop new organic transformations, ${ }^{[34-37]}$ we would like to report a highly efficient route for the synthesis of amidoalkylnaphthol derivatives by one pot multi component-coupling of $\beta$-naphthol, aldehydes, amides/urea catalyzed by commercially available, inexpensive, mild $\mathrm{Cu}\left(\mathrm{NO}_{3}\right)_{2} \cdot 3 \mathrm{H}_{2} \mathrm{O}$ as catalyst in good to excellent yields. (Scheme-1) 
<smiles>Oc1ccc2ccccc2c1</smiles>

1<smiles>[R]C=O</smiles><smiles>COC(=O)NOC(C)(C)C</smiles>

3<smiles>[R]C(=O)NC([R])c1c(O)ccc2ccccc12</smiles>

$(4 a-4 z)$

\section{Scheme-1}

\section{Experimental}

\section{MATERIALS AND METHODS}

Chemicals were purchased from Merck and Fluka and directly used for the synthesis. Thin layer chromatography (TLC): precoated silica gel plates (60 F254, 0.2mm layer; E. Merck). ${ }^{1} \mathrm{HNMR}$ (Avance $300 \mathrm{MHz}$ ) spectra were recorded in DMSO using TMS as internal standard. Melting points (m.p.) were determined on a Fischer-Johns melting point apparatus. IR and MS were recorded on a Thermo Nicolet Nexus 670 FT-IR Spectrometer and Finnegan MAT 1020 Mass spectrometer operating at $70 \mathrm{eV}$.

General procedure for the synthesis of amidoalkylnaphthol derivatives: A mixture of $\beta$-naphthol (1 mmol) aldehyde (1mmol) amides (1.2 mmol) or urea $(1.2 \mathrm{mmol})$ and $\mathrm{Cu}\left(\mathrm{NO}_{3}\right)_{2} \cdot 3 \mathrm{H}_{2} \mathrm{O}$ $(10 \mathrm{~mol} \%)$ in acetonitrile $(10 \mathrm{ml})$ was refluxed at $90^{\circ} \mathrm{C}$ for the time specified in (Table-3) after completion of the reaction as indicated by TLC, the reaction mixture was cooled to room temperature and washed with water and extracted with ethyl acetate, dried over $\mathrm{Na}_{2} \mathrm{SO}_{4}$ concentrated under vacuum and the crude mixture was purified by recrystalization from ethanol to afford pure product.

\section{Spectral data of the selected compounds:}

$N$-[phenyl-(2-hydroxy-naphthalen-1-yl) methyl]acetamide (4a): IR(KBr): 3406, 3250, 2920, 1640, 1583, 1519, 1437,1369, 1338, 1278, $1275,1190,1029,987,940,875,840,810,743$ $\mathrm{cm}^{-1}$; 1HNMR (300 MHz, DMSO-d6) $\delta=9.98$ (s, 1H), 8.45-8.42 (d, 1H), 7.84-7.74(m, 3H), 7.37-7.11 (m, 9H), 1.97 (s, 3H) ppm; MS(EI): $\mathrm{m} / \mathrm{z}(\%) 292(\mathrm{M}+)$.

N-[(4-Methyl phenyl)-(2-hydroxy-naphthalen-1-yl)methyl]acetamide (4b): IR (KBr): 3420 , $3320,3058,1621,1580,1561,1520,1466,1392$, 1298, 1202, 1145, 1051, 945, 890, 748, 745, 719 $\mathrm{cm}^{-1} ;{ }^{1} \mathrm{HNMR}\left(300 \mathrm{MHz}, \mathrm{DMSO}-\mathrm{d}_{6}\right): \delta=1.96$ (s, 3H), $2.21(\mathrm{~s}, 3 \mathrm{H}), 7.08-7.03(\mathrm{~m}, 5 \mathrm{H}), 7.19(\mathrm{~d}$, $J=8.8 \mathrm{~Hz}, 1 \mathrm{H}), 7.24$ (t, $J=7.1 \mathrm{~Hz}, 1 \mathrm{H}), 7.34$ (m, $1 \mathrm{H}), 7.74(\mathrm{~d}, J=8.8 \mathrm{~Hz}, 1 \mathrm{H}), 7.78(\mathrm{~d}, J=7.9 \mathrm{~Hz}$, 1H), 7.82 (br, 1H), 8.36 (d, $J=8.1 \mathrm{~Hz}, 1 \mathrm{H}), 9.91$ (s,1H) ppm; MS(EI): m/z (\%) $305\left(\mathrm{M}^{+}\right)$.

N-[(4-Methoxy phenyl)-(2-hydroxy-naphthalen-1-yl)methyl]acetamide (4c): IR(KBr): 3396 , 3078, 3002, 2967, 2832, 2787, 2704, 2614, $1627,1581,1515,1438,1378,1334,1304,1279$, $1268,1179,1088,1075,1043,983,930,880$, 850, 822, 814, 8035, $745 \mathrm{~cm}^{-1}$; ${ }^{1} \mathrm{HNMR}$ (300 MHz, DMSO-d6) $\delta=10(\mathrm{~s}, 1 \mathrm{H}), 8.4(\mathrm{~d}, 1 \mathrm{H})$, $7.80-7.73(\mathrm{~m}, 4 \mathrm{H}), 7.35-7.04(\mathrm{~m}, 6 \mathrm{H}), 2.5(\mathrm{~s}$, $3 \mathrm{H}), 1.953$ (s, 3H) ppm; MS(EI) m/z: $322\left(\mathrm{M}^{+}\right)$.

N-[4-Nitro phenyl)-(2-hydroxy-naphthalen1-yl)methyl]acetamide (4i): $\operatorname{IR}(\mathrm{KBr}): 3391$, $3072,1640,1602,1524,1439,1352,1281,1246$, 1167, 1066, 1093, 983, 935, 883, 855, 825, 734, $750 \mathrm{~cm}-1$; 1HNMR (300 MHz, DMSOd6) $\delta=10.11(\mathrm{~s}, 1 \mathrm{H}), 8.59-8.56(\mathrm{~d}, 1 \mathrm{H}), 8.14$ $-8.12(\mathrm{~m}, 2 \mathrm{H}), 7.83-7.79(\mathrm{~m}, 3 \mathrm{H}), 7.41-7.38$ $(\mathrm{m}, 3 \mathrm{H}), 7.28-7.16(\mathrm{~m}, 2 \mathrm{H}), 2.02(\mathrm{~s}, 3 \mathrm{H}) \mathrm{ppm}$; MS(EI) m/z: $337\left(\mathrm{M}^{+}\right)$.

N-[2-Hydroxy naphthalen-1-yl)-(naphthalen-1-yl)methyl]acetamide (4m): IR(KBr): 3420 3300, 2316, 1659, 1640, 1449, 1523, $748 \mathrm{Cm}^{-1}$; ${ }^{1} \mathrm{HNMR}\left(300 \mathrm{MHz}, \mathrm{DMSO}-\mathrm{d}_{6}\right): \delta=9.41(\mathrm{~s}, 1 \mathrm{H}$, $\mathrm{OH}), 8.52$ (d, J = 8.5 Hz, 1H), $8.12(\mathrm{~d}, \mathrm{~J}=8.1$ $\mathrm{Hz}, 1 \mathrm{H}), 7.81-7.85(\mathrm{~m}, 7 \mathrm{H}, \mathrm{Ar}-\mathrm{H}), 7.28-7.34$ (m, 6H, Ar-H), 2.88 (s, 3H, $\mathrm{CH}_{3}$ ) ppm; MS(EI) $\mathrm{m} / \mathrm{z}: 341\left(\mathrm{M}^{+}\right)$.

N-[3-Nitro phenyl)-(2-hydroxy-naphthalen1-yl)methyl]benzamide (4u): IR (KBr): 3369, 3278, 3099, 2978, 2956, 1640, 1578,1543, 1522 , 1521, 1480, 1439, 1347, 1308, 1280, 1207, 1171, 1093, 1070, 963, 934, 867, 820, $740 \mathrm{~cm}-1 ; 1 \mathrm{H}$ NMR (300 MHz, DMSO-d6) $\delta 10.42$ (s, 1H), 9.15-9.13 (d, 1H), 8.11-8.08 (m, 4H), 7.90- 7.82 (m, 5H), $7.73-7.71(\mathrm{~m}, 2 \mathrm{H}), 7.61-7.38(\mathrm{~m}, 6 \mathrm{H})$ ppm; MS(EI) m/z: $398\left(\mathrm{M}^{+}\right)$. 
Copper Nitrate Trihydrate Catalyzed Efficient One Pot Synthesis

$\mathrm{N}$-[4-Nitro phenyl)- (2-hydroxy-naphthalen1-yl)methyl]urea (4w) : IR(KBr): 3481, 3405, 3377, 3179, 3062, 2924, 2849, 1716, 1655, 1600, $1517,1439,1346,1257,1140,1109,1018,853$, $825,746 \mathrm{~cm}-1$; 1H NMR (300 MHz, DMSO-d6) $\delta 9.957(\mathrm{~s}, 1 \mathrm{H}), 7.82-7.73(\mathrm{~m}, 3 \mathrm{H}), 7.41-7.27$ (m, 1H), $7.24-7.11(\mathrm{~m}, 3 \mathrm{H}), 6.89(\mathrm{~m}, 2 \mathrm{H}), 6.75$ - $6.72(\mathrm{~m}, 3 \mathrm{H}), 5.82(\mathrm{~s}, 2 \mathrm{H}), 3.65$ (s, 3H) ppm; MS(EI): $\mathrm{m} / \mathrm{z}(\%) 322\left(\mathrm{M}^{+}\right)$.

\section{RESULTS AND DISCUSSION}

In the present study we extend the scope of the $\mathrm{Cu}\left(\mathrm{NO}_{3}\right)_{2} \cdot 3 \mathrm{H}_{2} \mathrm{O}$ catalyzed synthesis of amido alkylnaphthol derivatives and the results are presented here. In order to optimize the reaction conditions initially we studied the efficiency of $\mathrm{Cu}\left(\mathrm{NO}_{3}\right)_{2} \cdot 3 \mathrm{H}_{2} \mathrm{O}$ by taking catalytic amount of 10 $\%$ mol and benzaldehyde $(1 \mathrm{mmol}) \beta$-naphthol $(1 \mathrm{mmol})$ and acetamide $(1.2 \mathrm{mmol})$ in acetonitrile $(10 \mathrm{ml})$ as model reaction, the reaction gave the corresponding $\mathrm{N}$-[phenyl-(2-hydroxynaphthalen-1-yl) methyl]-acetamide with $92 \%$ yield in 4hours, with refluxing at $90^{\circ} \mathrm{C}$ (Table-3). In the absence of $\mathrm{Cu}\left(\mathrm{NO}_{3}\right)_{2} \cdot 3 \mathrm{H}_{2} \mathrm{O}$ even up to $6 \mathrm{~h}$ no reaction was observed although the amount of catalyst has been optimized to $10 \% \mathrm{~mol},(5 \%$ mol) also worked when longer reaction times were employed, while screening of various catalysts we found that $\mathrm{Cu}\left(\mathrm{NO}_{3}\right)_{2} \cdot 3 \mathrm{H}_{2} \mathrm{O}$ was more effective than other nitrates tested in terms of isolated yields (92\%) (10 Table-1), we choose $\mathrm{Cu}\left(\mathrm{NO}_{3}\right)_{2} \cdot 3 \mathrm{H}_{2} \mathrm{O}$ as the suitable catalyst for further reactions due to its easy availability, cost effectiveness, easy handling intrigued by these observations we have then tested the efficiancy of several copper salts such as $\mathrm{Cu}(\mathrm{OAc})_{2}, \mathrm{Cu}(\mathrm{Cl}$, $\mathrm{Br}$, I) along with $\mathrm{Cu}\left(\mathrm{NO}_{3}\right)_{2} \cdot 3 \mathrm{H}_{2} \mathrm{O}$ on the model reaction and among the copper salts screened $\mathrm{Cu}\left(\mathrm{NO}_{3}\right)_{2} \cdot 3 \mathrm{H}_{2} \mathrm{O}$ was found to be best both in terms of reaction time and yields. The model reaction was performed in various solvents using $\mathrm{Cu}\left(\mathrm{NO}_{3}\right)_{2} \cdot 3 \mathrm{H}_{2} \mathrm{O}$ as the catalyst to identify the best condhtions for the reaction. A range of solvents such as $\mathrm{CHCl}_{3}$, DCM, THF, DMSO, toluene, $\mathrm{H}_{2} \mathrm{O}$ and acetonitrile were examined and acetonitrile emerged as the solvent of choice in terms of reaction kinetics and product yields (Table-2). Encouraged by the results obtained for benzaldehyde we generalized the reaction scope for a number of other aromatic functionalities chloro, fluoro, methyl, methoxy nitro were tolerated and gave good yields.

Table- 1: Screening of various catalysts (metal nitrates) $(10 \% \mathrm{~mol})$ on model reaction between $\beta$-naphthol (1 mmol) benzaldehyde $(1 \mathrm{mmol})$, acetamide $(1.2 \mathrm{mmol})$, in acetonitrile $(10 \mathrm{ml})$ heating at $90{ }^{\circ} \mathrm{C}$.

\begin{tabular}{cclc}
\hline Entry & Catalyst & \multicolumn{2}{c}{ Time (h) } \\
1 & $\mathrm{Ce}(\mathrm{NH} 4) 2 .(\mathrm{NO} 3) 6$ & 6 & 45 \\
2 & $\mathrm{Ni}(\mathrm{NO} 3) 3.6 \mathrm{H} 2 \mathrm{O}$ & 5.5 & 50 \\
3 & $\mathrm{Al}(\mathrm{NO} 3) 3.9 \mathrm{H} 2 \mathrm{O}$ & 6 & 50 \\
5 & $\mathrm{Zn}(\mathrm{NO} 3) 2.6 \mathrm{H} 2 \mathrm{O}$ & 5 & 60 \\
6 & $\mathrm{AgNO} 3$ & 5 & 50 \\
7 & $\mathrm{Fe}(\mathrm{NO} 3) 3.9 \mathrm{H} 2 \mathrm{O}$ & 5.5 & 60 \\
8 & $\mathrm{Rh}(\mathrm{NO} 3) 3.2 \mathrm{H} 2 \mathrm{O}$ & 5 & 76 \\
9 & $\mathrm{La}(\mathrm{NO} 3) 3.6 \mathrm{H} 2 \mathrm{O}$ & 5.5 & 65 \\
10 & $\mathrm{Cu}(\mathrm{NO} 3) 2.3 \mathrm{H} 2 \mathrm{O}$ & 4 & 92 \\
\hline
\end{tabular}

a = Isolated yields

Table- 2: Screening of various solvents $(10 \mathrm{ml})$ on model reaction between $\beta$-naphthol $(1 \mathrm{mmol})$ benzaldehyde (1mmol), acetamide (1.2 mmol), in the presence of $\mathrm{Cu}\left(\mathrm{NO}_{3}\right)_{2} 3 \mathrm{H}_{2} \mathrm{O}$ $(10 \mathrm{~mol} \%)$ heating at $90^{\circ} \mathrm{C}$.

\begin{tabular}{cccc}
\hline Entry & Solvent & Time (h) & Yield (\%)a \\
\hline 1 & DCM & 5.5 & 40 \\
2 & DMF & 6 & 60 \\
3 & THF & 6 & 55 \\
4 & H2O & 7 & 40 \\
5 & CH3CN & 4 & 92 \\
6 & EtOAc & 5 & 54 \\
7 & CHCl3 & 5 & 70 \\
8 & DEA & 5 & 68 \\
9 & Ethanol & 5.5 & 55 \\
\hline
\end{tabular}

$\mathbf{a}=$ Isolated yields

Aliphatic aldehydes such as butyraldehyde, propanaldehyde (Table-3) the reaction gave the corresponding amidoalkynaphthol derivatves in 
good yield. In case of hereoaromatic aldehyde we have isolated the product with impurity (Table-3). Aromatic aldehydes carrying either electron-withdrawing or electron-donating groups reacted successfully and gave the desired product in high yields. A possible mechanism for this transformation is proposed in the literature, ${ }^{[7,10,}$ ${ }^{19,29,32]}$ reaction of $\beta$-naphthol with aldehydes in the presence of lewis acid catalyst is known to give ortho-quinone methides $(o-\mathrm{QMs})$. The $(o-$ QMs) generated insitu, have been reacted with amides via conjugate addition to form 1-amidoalkyl-2-naphthol derivatives (Table-3). On the other hand, the reaction with amine such as aniline was utilized and no amino alkyl naphthols were obtained. ( Scheme-2)

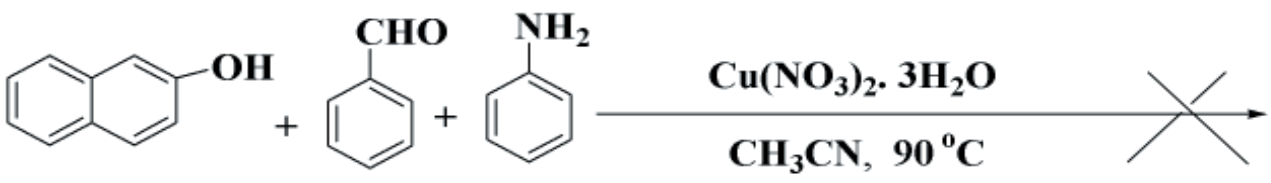

Scheme-2

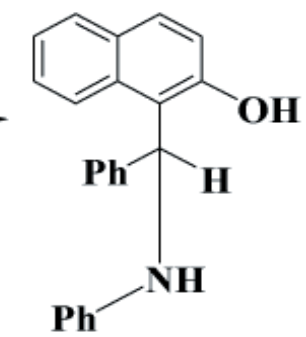

Table-3 Synthesis of amidoalkyl naphthols in the presence of Cupric Nitrate Trihydrate (10mol\%)

\begin{tabular}{|c|c|c|c|c|c|c|c|}
\hline Entry & $\mathbf{R}^{1}$ & $\mathbf{R}^{2}$ & Product $^{\mathrm{a}}$ & Time (h) ${ }^{\mathrm{c}}$ & Yield $^{\mathrm{b}}(\%)$ & m.p. & [Ref] \\
\hline 1 & $\mathrm{C}_{6} \mathrm{H}_{5}$ & $\mathrm{CH}_{3}$ & $4 a$ & 4 & 92 & $242-244$ & 32 \\
\hline 2 & $4-\mathrm{CH}_{3} \mathrm{C}_{6} \mathrm{H}_{4}$ & $\mathbf{C H}_{3}$ & $4 b$ & 4 & 90 & $222-223$ & 32 \\
\hline 3 & 4- $\mathrm{OCH}_{3} \mathrm{C}_{6} \mathrm{H}_{4}$ & $\mathbf{C H}_{3}$ & $4 c$ & 4 & 90 & $183-184$ & 32 \\
\hline 4 & $2,4-\mathrm{Cl}_{2} \mathrm{C}_{6} \mathrm{H}_{3}$ & $\mathbf{C H}_{3}$ & $4 d$ & 4.5 & 85 & $202-204$ & 32 \\
\hline 5 & $4-\mathrm{ClC}_{6} \mathrm{H}_{4}$ & $\mathrm{CH}_{3}$ & $4 e$ & 4.5 & 89 & $237-239$ & 33 \\
\hline 6 & $4-\mathrm{N}\left(\mathrm{CH}_{3}\right)_{2} \mathrm{C}_{6} \mathrm{H}_{4}$ & $\mathbf{C H}_{3}$ & $4 f$ & 4.5 & 82 & $120-123$ & 32 \\
\hline 7 & $\mathrm{C}_{6} \mathrm{H}_{4} \mathrm{~N}$ & $\mathrm{CH}_{3}$ & $4 g$ & 4.5 & 63 & 193-194 & 31 \\
\hline 8 & $2-\mathrm{HOC}_{6} \mathrm{H}_{4}$ & $\mathrm{CH}_{3}$ & $4 h$ & 4 & 80 & 216-218 & 31 \\
\hline 9 & $4-\mathrm{NO}_{2} \mathrm{C}_{6} \mathrm{H}_{4}$ & $\mathrm{CH}_{3}$ & $4 i$ & 4 & 92 & $251-253$ & 33 \\
\hline 10 & $\mathrm{C}_{6} \mathrm{H}_{4}-\mathrm{CH}=\mathrm{CH}$ & $\mathrm{CH}_{3}$ & $4 j$ & 4.5 & 75 & $185-188$ & 31 \\
\hline 11 & $2-\mathrm{ClC}_{6} \mathrm{H}_{4}$ & $\mathrm{CH}_{3}$ & $4 k$ & 4.5 & 80 & 213-214 & 32 \\
\hline 12 & $4-\mathrm{FC}_{6} \mathrm{H}_{4}$ & $\mathrm{CH}_{3}$ & 41 & 4.5 & 90 & 229-231 & 32 \\
\hline 13 & $\mathrm{C}_{10} \mathrm{H}_{7}$ & $\mathrm{CH}_{3}$ & $4 m$ & 4.5 & 80 & $228-230$ & 32 \\
\hline 14 & 3-OH4- $\mathrm{CH}_{3} \mathrm{OC}_{6} \mathrm{H}_{3}$ & $\mathrm{CH}_{3}$ & $4 n$ & 4 & 83 & $237-239$ & 32 \\
\hline 15 & $\mathrm{CH}_{3}-\mathrm{CH}_{2}-\mathrm{CH}_{2}$ & $\mathrm{CH}_{3}$ & 40 & 5 & 78 & 224-227 & 32 \\
\hline $\begin{array}{l}16 \\
17\end{array}$ & $\begin{array}{l}\underset{\mathbf{C}_{\mathbf{6}} \mathrm{H}_{5}}{\mathrm{CH}_{3}-\mathrm{CH}_{2}} \\
\end{array}$ & $\begin{array}{c}\mathrm{CH}_{3} \\
\mathrm{C}_{6} \mathrm{H}_{5}\end{array}$ & $\begin{array}{l}4 p \\
4 q\end{array}$ & $4^{5}$ & $\begin{array}{l}75 \\
92\end{array}$ & $\begin{array}{c}178-180 \\
\mathbf{2 3 8 - 2 4 0}\end{array}$ & $\begin{array}{l}32 \\
32\end{array}$ \\
\hline 18 & 4-NO2 $\mathrm{C}_{8} \mathrm{H}_{4}$ & $\mathrm{C}_{\mathbf{d}} \mathrm{H}_{5}$ & $4 \mathbf{r}$ & 4 & 90 & $240-242$ & $\mathbf{3 3}$ \\
\hline 19 & $4 \mathrm{CH}_{3} \mathrm{OC}_{4} \mathrm{H}_{4}$ & $\mathrm{C}_{\mathrm{d}} \mathrm{H}_{5}$ & $4 s$ & 4 & 90 & 209-212 & $\mathbf{3 2}$ \\
\hline 2n & $2-\operatorname{CrC}_{4} \mathrm{H}_{4}$ & $\mathrm{C}_{\mathrm{d}} \mathrm{H}_{5}$ & 4t & 4.5 & 85 & $286-288$ & $\mathbf{3 z}$ \\
\hline 21 & $\mathrm{HNO}_{2} \mathrm{C}_{4} \mathrm{H}_{4}$ & $\mathrm{C}_{d} \mathrm{H}_{5}$ & 40 & 4.5 & 89 & 233-235 & $\mathbf{3 3}$ \\
\hline $\mathbf{2 2}$ & $\mathrm{C}_{\mathbf{s}} \mathrm{H}_{5}$ & $\mathbf{N H}_{2}$ & $4 w$ & 4 & $\mathbf{z z}$ & 179-181 & 32 \\
\hline $\mathbf{2 3}$ & $4 \mathrm{NO}_{2} \mathrm{C}_{8} \mathrm{H}_{4}$ & $\mathbf{N H}_{2}$ & 4w & 4 & 79 & 16:-165 & 33 \\
\hline 24 & $3-\mathrm{CH}_{3} \mathrm{OCC}_{6} \mathrm{H}_{4}$ & $\mathbf{F H}_{2}$ & $4 x$ & 4 & $\mathbf{8 0}$ & 243-245 & $\mathbf{3 3}$ \\
\hline 25 & $\mathrm{BNO}_{2} \mathrm{C}_{4} \mathrm{H}_{4}$ & $\mathbf{N H}_{\mathbf{z}}$ & $4 y$ & 4 & 92 & 191-193 & 33 \\
\hline 26 & $4-\mathrm{CH}_{3} \mathrm{C}_{8} \mathrm{H}_{4}$ & $\mathbf{N H}_{2}$ & $4 x$ & 4.5 & 88 & $115-118$ & $\mathbf{3 3}$ \\
\hline
\end{tabular}

$\mathrm{a}=$ All the products are characterized by spectral analysis, $\mathrm{b}=$ Isolated yields , $\mathrm{c}=$ reflux at $90^{\circ}$ 


\section{CONCLUSION:}

Copper Nitrate Trihydrate Catalyzed Efficient One Pot Synthesis

We have developed a practical and new, general efficient procedure for the one pot synthesis of amidoalkylnaphthol derivatives by coupling of various aromatic aldehydes with amides urea and $\beta$-naphthol using $\mathrm{Cu}\left(\mathrm{NO}_{3}\right)_{2} \cdot 3 \mathrm{H}_{2} \mathrm{O}$ as catalyst. The present protocol has several advantages of readily available, inexpensive catalyst, mild reaction conditions, easy handling, excellent yields, greater selectivity, operational and experimental simplicity. We believe that $\mathrm{Cu}\left(\mathrm{NO}_{3}\right)_{2} \cdot 3 \mathrm{H}_{2} \mathrm{O}$ catalyzed methodology will definitely be a valuable addition to the existing process in the field of amidoalkylnaphthol derivatives.

\section{REFERENCES:}

1. (a) Ugi I, Pure Appl. Chem. (73), 187, (2001), and references cited therein; (b) Devi. I, Bhuyan. P. J, Tetrahedron Lett. (45), 8625 (2004) (c)For a monograph, see: Zhu,J., Bienayme, H., Eds. Multi component Reactions; Wiley-VCH,: Weinheim, Germany, (2005) (d) Domling, A.Chem.Rev (106) 17 (2006) (e) D'Souza, D. M, Mueller, T. J.J. Chem. Soc. Rev. (36) 3169 (2007) (f) Cariou. C.C.A,Clarkson, G. J, Shipman, M J.Org. Chem. (73) 9762 (2008) (g) Alizadeh. A, Mobahedi. F, Esmaili. A, Tetrahedron Lett. 2006, 47, 4469; (h) Umkeherer, M.; Kalinski, C.; Kolb, J.; Burdack, C. Tetrahedron Lett..(47), 2391(2006).

2. a)Ramon, D. J.; Yus, M. Angew. Chem., Int. Ed. (44) 1602 (2005) b) Tejedor, D, Garcia-T.F, Chem. Soc. Rev. (36) 484 (2007).

3. Domling, A.; Ugi, I.Angew. Chem. Int. Ed. 39 (18), 3168 (2000).

4. (a) Seebach. D, Matthews J. L. J.Chem. Soc., Chem. Commun. 2015 (1997), (b) Wang.Y-F, Izawa. T, Kobayashi. S, Ohno. M, J. Am. Chem. Soc. (104) 6465 (1982), (c) Knapp, S.Chem. Rev. (95) 1859 (1995) (d) Juaristi, E. In Enantioselective Synthesis of $\beta$-Amino Acids; John Wiley \& Sons: New York, (1997), (e) Seebach, D, Matthews, J. L, Chem. Commun. 2015, (1997).

5.(a) Dingermann T, Steinhilber, D, Folkers, G. Molecular Biology in Medicinal Chemistry; WileyVCH, Weinheim, (2004), (b) Shen, A. Y, Tsai C. T, Chen C. L, Eur. J. Med. Chem.(34), 877 (1999) (c) Shen A. Y, Chen, C. L, Lin. C. I, Chin. J. Physiol. (35) 45 (1992).

6. Hulst. R, Heres H, Peper N. C. M. W, Kellogg R. M, Tetrahedron: Asymmetry (7)1373 (1996) (b) Li, X, Yeung, C.-H, Chan A. S. C, Yang, T.-K. Tetrahedron: Asymmetry (10), 759 (1999).
7. Kantevari S, Vuppalapati S V N and Nagarapu L, Catal Commun., (8), 1857(2007).

8. Selvam N.P, Perumal P.T, Tetrahedron Lett., (47), 7481(2006).

9. Nagarapu L, Baseeruddin M, Apuri S and Kantevari S, Catal Commun., (8), 1729 (2007)

10. Khodaei M. M, Khosropour A. R and Moghanian H, Synlett., (6), 916 (2006) .

11. Patil S.B, Singh P.R, Surpur M.P and Samant S.D, Ultrason Sonochem.,(14), 515 (2007).

12. (a) Hajipour A. R, Ghayeb Y, Sheikhan N and Ruoho A.E, Tetrahedron Lett., (50), 5649,(2009). (b) Kotadia D. A, and Soni S. S, J Mol Catal Chem., (353), 44( 2012) (c) Luo J and Zhang Q, Monatsh Chem., 142(9), 923 (2011).

13. Patil S.B, Singh P. R, Surpur M. P, and Samant S. D, Synth Commun., (37), 1659 (2007).

14. Shaterian H.R, Amirzadeh A, Khorami F and Ghashang M, Synth Commun., (38), 2983 (2008).

15. Shaterian H. R, Yarahmadi $H$ and Ghashang $M$, Bioorg Med Chem Lett, .,(18), 788 (2008).

16. Kumar A, Rao M. S, Ahmad I and Khungar B, Can J Chem., (87), 714 (2009).

17. Mahdavinia G.H, Bigdeli M. A, Chin Chem Lett., (20), 383 (2009).

18. Shaterian H.R, Khorami F, Amirzadeh A and Ghashang M, Chin J Chem., (27), 815 (2009).

19. Datta B and Pasha M. A, Ultrason Sonochem., 18(2), 624 (2011).

20. Chavana N. L, Naika P. N, Nayakb S. K and Kusurkar R. S, Synth Commun., (40), 2941 (2010).

21. Su W. K, Tang W. Y and Li J. J, J Chem Res., 123 (2008).

22. Nandi G.C, Samai S, Kumar R and Singh M. S, Tetrahedron Lett., (50) 7220 (2009).

23. Hamid R. S, Hossein Y, and Majid G, Turk J Chem; (33) 449 (2009) .

24. Supale A. R and Gokavi G. S, J Chem Sci., 122(2), 189 (2010).

25. Sunil R. M, Rikesh S. J, and Kalpana C. M, J. Chem. Sci. 123(4), 427 (2011).

26. Ghorbani-Vaghei R and Malaekehpour S. M, Eur J Chem., (8), 1086 (2010).

27. Xiao-hua CAI , International Journal of Chemistry,. 3(1), 119 (2011).

28. Khazaeia A, Zolfigol M. A, Moosavi-Zare A. R, Zare A, Parhami A and Khalafi- Nezhad A, Appl Catal A Gen., (386), 179 (2010).

29. Wang M, Liang Y, Zhang T. T, and Gao J. J, Chin Chem Lett., (23), 65 (2012). 
30. Zahed K. J, and Hadi F, Bull. Chem. Soc. Ethiop. 26(3), 473( 2012).

31. Mohammad Ali A, Bi Bi F. M, Hamideh E, J.chem.sci., 125 ( 3) 561 (2013).

32. Javad Safari, Journal of Molecular Catalysis A: Chemical (379), 269 (2013).

33. Nourallah. H, Malek T. M, Sayyed M. H.K, Jasemaboonajmi and M. Safarzaei, Chem Sci Trans., 2(S1), S330 (2013).

34. Nasreen A. Tetrahedron Lett. (54), 3797 (2013).

35. Nasreen A. Asian journal of chemistry, (25), 7535 (2013).

36. a) Varala R, Nasreen A, Ramu E, Adapa, S. R.
Tetrahedron Lett. (48), 69 (2007) b) Varala R, Nasreen A, Adapa S. R. Can. J. Chem., (85) 1 (2007) c) Nasreen A, Varala R, Adapa, S. R. J. Heterocycl. Chem. (44), 1 (2007).

37. Aayesha Nasreen, Rita M. Borik, Oriental Journal of Chemistry 30(4), (2014) in press.

38. Durgareddy G. K, Ravikumar R, Ravi S, Adapa S. R J. Chem. Sci. (125),75 (2013).

39. Aayesha Nasreen, and Srinivas R . Adapa, Heterocyclic Comminications, (5), 501 (2001).

40. Aayesha Nasreen, and Srinivas R. Adapa, Organic preparations and Procedures International, (32), 373 (2000). 